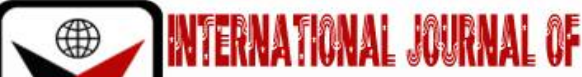

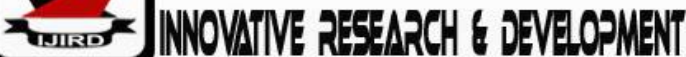

ISSN 2278-0211 (Online)

\section{The Protective Role of Self-Care in the Development of Compassion Fatigue among Health Care Professionals in Moi Teaching and Referral Hospital, Kenya}

Kariuki Jane Njeri
Principal Counselor, Department of Psychological Counseling,
Moi Teaching \& Referral Hospital, Kenya
Dr. Scholastic Adeli
Senior Lecturer, Department of Sociology, Psychology \& Anthropology
Moi University, Kenya
Kimani Chege Gabriel
Associate Professor, Department of Sociology, Psychology \& Anthropology
Moi University, Kenya

\begin{abstract}
:
Compassion fatigue is a condition unique to the human service occupations, characterized by a state of tension and preoccupation with the traumatized clients by re-experiencing traumatic events. With increased incidences of traumatic events both nationally and globally, the greater burden of care and after-care is usually borne by health care professionals. The objective of this study was to determine the protective role of self-care in the development of compassion fatigue among health care professionals in Moi Teaching and Referral Hospital (MTRH) Eldoret, Kenya. The study was guided by Figley Model of Compassion Fatigue and adopted the Ex Post Facto research design. The target population was 76 doctors, 212 nurses and 33 counselors working in twelve units (grouped into more traumatizing and less traumatizing) offering specialized patient care services. A stratified simple random sampling technique was used to select a sample of 82 participants comprising of 19 Doctors, 54 Nurses and 9 Counselors. The independent variable was Years of Experience while Compassion Fatigue was the dependent variable. Data was collected using a demographic questionnaire, the 'Professional Quality of Life Scale (ProQoL) version V'. Data was analyzed using the Pearson correlation $r$ at $\alpha=0.05$ level of significance and data presented in form of percentages, frequencies and means while graphic presentation was in form of tables. Since $p<0.005$ in the Pearson correlation statistic table, the study hypothesis was rejected. The consequent implication was that the lesser the self-care activities engagement, the higher the level of compassion fatigue among the health care professionals. Based on the findings of this study, it is recommended that regular training on self-care that encompasses all the six subsets of self-care (Physical, Spiritual, Emotional, Psychological, and Workplace Balance) should be introduced in the work place as it will help in both preventing compassion fatigue and managing it whenever it occurs.
\end{abstract}

Keywords: Compassion fatigue, self-care

\section{The Study Problem}

Kenya in the recent past has been experiencing a myriad of traumatic incidences which are increasing by the day. Critical incidences are such as domestic violence, bizarre murders, rape and kidnapping, collapsing of buildings and terrorist attacks that has been experienced in Nairobi, Lamu, Mandera, Wajir and the attack of Garrisa University College where 147 students lost their lives and scores of others suffered both physical and psychological trauma. The country is also facing an upsurge of both social and medical problems such as child delinquency, drug addiction, pornography and chronic illnesses such as cancer and HIV/AIDs. Effects of all these and many other health related issues expose health care professionals to compassion fatigue.

As a referral facility, and due to its location on the highway to Uganda, Rwanda, Burundi and South Sudan, Moi Teaching and Referral Hospital (MTRH) handles emergencies of all nature from Western Kenya Regions and beyond. Health care professionals in the institution are therefore always on high alert because of the risks of exposure to highly infectious diseases such as Ebola and Multi-Drug Resistance Tuberculosis (MDR), among other dreadful conditions. Further, survivors of critical incidents such as the 2007/8 Post Election Violence (PEV) that affected the country are usually attended to at MTRH. The health care professionals not only attended to the hospitalized but also extended care to the survivors in the camps which further exposed them to traumatic experiences. 
Prevalence of Compassion Fatigue among health professionals in other parts of the world has been widely studied (Smit, 2006). However, limited studies have been documented in Kenyan hospitals. Specifically, no study has attempted to carry out an investigation of compassion fatigue on more than one health care profession in one setting and more importantly drawing a comparison of the constructs among doctors, nurses and counselors in MTRH despite the widespread evidence of compassion fatigue in this population globally. This study therefore envisaged filling this gap by investigating the protective role played by self-care in the management of compassion fatigue amongst health care professionals in MTRH.

\section{The Study Hypothesis} variables: -

The following hypothesis was tested to determine the association between the independent and dependent

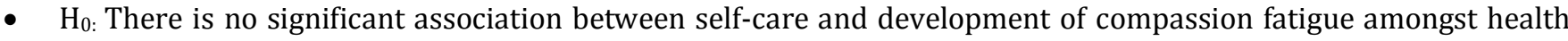
care professionals in MTRH

\section{Research Design}

The study adapted an ex post facto research design. This design was ideal since the current study aimed at describing and establishing the relationship between predictive and protective factors and the development of compassion fatigue among Doctors, Nurses and Counselors. Ex post facto design describes an existing relationship between variables which cannot be manipulated at the time of the study and whose difference has already occurred and must be studied in retrospect. It comprises of collecting data to determine the cause, or reason for preexisting differences in groups of individuals (Fraenkeal \& Wallen, 2010). The design therefore allowed investigation of the difference in the nature of association of work experience and the development of compassion fatigue among Doctors, Nurses and Counselors in MTRH hence allowing for a comparison of compassion fatigue between the three categories of health care professionals.

\section{Target Population}

The target population for this study comprised of 76 Doctors, 212 Nurses and 33 Counselors working in a total of 12 units offering specialized patient care in Moi Teaching and Referral Hospital - Eldoret. This population was based on the staff population records maintained by the Human Resource Department of the Hospital. The units were Intensive Care Unit (ICU)/High Dependence Unit (HDU), Sexual and Gender Based Violence Centre, Oncology Unit, New Born Unit (NBU), Ophthalmology (Eye) Unit and Alcohol and Drug Rehabilitation Unit (ADA-R), Psychiatric unit, Accident and Emergency Department, Burns Unit, Cardiac Care Unit, Renal Unit and Labour Ward. A list of the health care professionals (doctors, nurses and counselors) working in the twelve units was obtained from the Chief Nurse Office, the office of the Deputy Director Clinical Services and the Head of Department of Psychological Counseling respectively. The researcher ensured that the sampling frame to be used for the study (the list of Doctors, Nurses and Counselors working in the twelve units) was complete and accurate by confirming the same against records kept by the Officers-in-charge of these units so that the findings from the study would be generalized beyond the sample or the sampling frame from which the sample was drawn. Distribution of the target population is presented in Table 1.

\begin{tabular}{|c|c|}
\hline & Total Number \\
\hline Doctors & 76 \\
\hline Nurses & 212 \\
\hline Counselors & 33 \\
\hline Total & 321 \\
\hline
\end{tabular}

Table 1: Distribution of Target Population Health Care Professional

\section{Sample Size and Sampling Technique}

The coefficient of variation formula by Nassiuma (2000) was used to determine the sample size. This formula is useful in obtaining samples from population whose underlying probability distributions are unknown. A coefficient of variation of $21 \%$ and standard error of $2 \%$ was used in this study. The lower limit for coefficient of variation and standard error was selected so as to ensure low variability in the sample and minimize the degree of error (Ndung'u, 2008).

$$
n=\frac{N C V^{2}}{C V^{2}+(N-1) e^{2}}
$$

Where,

$\mathrm{n}=$ Sample size

$\mathrm{N}=$ Population

$\mathrm{CV}=$ Coefficient of Variation

e $=$ error

Therefore $-\mathrm{N}=321 ; \mathrm{CV}=21 \%$; $=2 \%$

$$
\begin{gathered}
\frac{321 \times 0.0441}{0.0441+(320 \times 0.0004)} \\
\frac{14.1561}{0.1721}=82.3=82
\end{gathered}
$$

The sampling procedure for this study was stratified simple random technique where the obtained sample size of 82 was proportionately distributed among the three-health care professional categories (strata) as shown in Table 2. 


\begin{tabular}{|c|c|c|}
\hline HCP & Target Population & Sample size \\
\hline Doctors & 76 & 19 \\
\hline Nurses & 212 & 54 \\
\hline Counselors & 33 & 9 \\
\hline Total & $321(\mathrm{~N})$ & $82(\mathrm{n})$ \\
\hline
\end{tabular}

Table 2: Sample Distribution of Health Care Professionals (Hcp) by Category

The final distribution of the sample from the various work settings is as shown in table 3 below.

\begin{tabular}{|c|c|c|c|}
\hline Work Settings & Doctor & Nurses & Counselors \\
\hline ICU & 1 & 7 & 1 \\
\hline CAR-E & 1 & 1 & 1 \\
\hline Oncology & 3 & 3 & 1 \\
\hline New born & 2 & 9 & 1 \\
\hline Ophthalmology & 1 & 3 & - \\
\hline ADA-R & 1 & 2 & 1 \\
\hline Psychiatry & 2 & 3 & 1 \\
\hline A\&E & 2 & 3 & 1 \\
\hline Burns Unit & 1 & 3 & 1 \\
\hline Cardiac Care & 1 & 4 & - \\
\hline Renal Unit & 1 & 6 & - \\
\hline Labour Ward & 3 & 54 & 9 \\
\hline Total (N) & 19 & 3 & \\
\hline
\end{tabular}

Table 3: Sample Distribution of Health Care Professionals by Work Setting $\mathrm{N}=82$

A simple random sampling procedure was used to identify the participants from the obtained samples.

\section{Data Sources and Instruments}

Data for this study was collected using self-administered questionnaires. The questionnaires were organized in three parts. Part I was designed to gather demographic information about the respondents, part II, the Professional Quality of Life Scale version V (ProQoL- 5) was used to measure compassion fatigue.

The Professional Quality of Life Scale (ProQoL-5) Version V' is a standardized scale widely used to assess compassion fatigue and compassion satisfaction among helping professions. Developed by Stamm (2009), ProQoL-5, is a 30-item instrument that asks participants to respond based on how they have been feeling over the last 30 days on a 5-point Likert scale ( 1 being never, 5 being very often). The tool has been widely used in studies on compassion fatigue. The Demographic Questionnaire was developed by the researchers.

\section{Ethical Considerations}

This research involved collecting data from doctors, nurses and counselors using self-administered questionnaires. The research approval was obtained from the Institutional Ethics Committee (IREC), Moi University/MTRH and the National Commission for Science, Technology and Innovation (NACOSTI).

To meet the requirements of Ethical Principles of research dealing with human subjects and to ensure confidentiality of the participants, the following measures were undertaken:

- The objectives, issues, risks and benefits of the study were conveyed to the participants.

- Formal consent was obtained from the participants prior to administration of the questionnaires.

- The participants who agreed to participate were informed that they were free to withdraw from the study at any time.

- The privacy of the participants and the confidentiality of data that was obtained from the participants was strictly maintained in such a manner that the participants cannot be identified in the report or any related publications.

\section{Data and Results}

\subsection{Association between Self-Care and Compassion Fatigue}

The study hypothesis stated and tested for self-care and Compassion Fatigue was

- $\quad \mathrm{H}_{0}$ : There is no significant association between self-care and development of compassion fatigue amongst health care professionals in Moi Teaching and Referral Hospital

Self-Care, a protective factor of compassion fatigue was measured using Self-Care Assessment Worksheet (SCAW) whose primary purpose is to determine the participants overall engagement in Self-Care activities. Engagement in selfcare has been shown to considerably ameliorate development of compassion fatigue among health care professionals (Star, 2013). Scores on self-care can range between 70 and 350 (Saakvitne \&Pearlman, 1996) and are divided into 6 subgroups thus, physical, psychological, emotional, spiritual, work and balance. Fewer scores denote higher involvement in self-care while high scores are an indication of low involvement in self-care activities. 
In the current study, participants total scores ranged between 80 and 236. These scores show the amount of self-care engaged by the participants for each of the self-care category as shown in Table 4.

\begin{tabular}{|c|c|c|c|c|c|c|}
\hline & $\begin{array}{c}\text { Physical } \\
\text { Self-care }\end{array}$ & $\begin{array}{c}\text { Psychological } \\
\text { Self-care }\end{array}$ & $\begin{array}{c}\text { Emotional } \\
\text { Self-care }\end{array}$ & $\begin{array}{c}\text { Spiritual } \\
\text { Self-care }\end{array}$ & $\begin{array}{c}\text { Workplace } \\
\text { Self-care }\end{array}$ & $\begin{array}{c}\text { Balance } \\
\text { Self-care }\end{array}$ \\
\hline Mean & 29.55 & 31.29 & 20.97 & 33.68 & 20.80 & 4.55 \\
\hline SD & 9.030 & 8.256 & 6.806 & 14.823 & 7.288 & 1.825 \\
\hline Min & 15 & 14 & 11 & 17 & 11 & 2 \\
\hline Max & 53 & 46 & 41 & 75 & 41 & 9 \\
\hline
\end{tabular}

Table 4: Overall Involvement in Self-Care among Health Care Professionals

First, the physical self-care subscale has a total of 15 items and the scores range from 15 to 75 . In the current study, participants' physical self-care scores ranged between 15 and 53 with a mean score of 29.55 and a standard deviation of 9.030. With these results (mean $=29.55$ and SD $=9.03$ and a range of $15-53$ ) the health care professionals were found to engage in physical self-care activities but only at an average level. This is considering their mean score in comparison to the maximum expected score of 53.

Secondly, the psychological scale has 13 items with a range of 13 to 65. Participants scored between 14 and 46 with a mean of 31.29 and a standard deviation of 8.256 indicating a lesser engagement in psychological self-care. In the third category, participants were asked to respond to the level of their emotional self-care engagement. The subscale contained 11 items with a score range of 11 to 55. Results indicate that participants' scores ranged from 11 to 41 with a mean score of 20.97 and a standard deviation of 6.806. Most of the health care professionals were therefore found to take care of their emotional needs but only averagely considering that the mean score for all participants was 20.97 for this subscale.

The fourth subscale of spiritual self-care has 17 items and its scores can range from 17 to 85 . Participants' scores in the current study ranged from 17 to 75 with a mean score of 33.68 and a standard deviation of 14.823. Despite the results indicating an average spiritual engagement with a mean score of 33.68 , there seems to be a disparity in the scores suggested by a relatively high standard deviation of about 15.

The next self-care subscale was on workplace which has 11 items. In this subscale scores can range from 12 to 60 . Just as the findings from the other self-care subsets in this study participants depicted a moderate workplace self-care as supported by a mean score of 20.80 and a standard deviation of 7.288 with a range of 11 to 41 . Lastly, the balance subscale contains 2 items and a score range between 2 and 10. Participants scored between 2 and 9, a mean of 4.55 and a standard deviation of 1.825, similarly suggesting an average level of a balance in self-care engagement of work-life, family, relationship, play and rest.

The general outlook of the results across all categories indicates that there is slightly above average self-care involvement across all self-care categories among the health care professionals. This in essence indicates that health care professionals utilize self-care which is a key factor in the reduction of compassion fatigue.

\begin{tabular}{|c|c|c|c|}
\hline & & Compassion Fatigue & Self-care \\
\hline \multirow{3}{*}{ Compassion Fatigue } & Pearson Correlation & 1 & $-.766^{* *}$ \\
\cline { 2 - 4 } & Sig. (2-tailed) & & .000 \\
\cline { 2 - 4 } & $\mathrm{N}$ & 75 & 75 \\
\hline
\end{tabular}

Table 5: Correlation of Self-Care and Compassion Fatigue

These descriptive results of association between self-care and compassion fatigue were further supported by the Pearson correlation analysis that revealed a significant negative correlation between compassion fatigue and self-care engagement at $r=-0.766, p=0.001$ as shown in Table 5. The negative correlation indicates that the higher the self-care scores the lesser the self-care activities engagement by the health care profession. Consequently, the lesser the self-care activities engagement, the higher the level of compassion fatigue among the health care professionals.

The study hypothesis was therefore rejected on the strength of the variable self-care having $\mathrm{p}<0.005$ (Table 5). Similar findings were reported in studies by (Bakibinga, 2012; Star, 2013) where negative correlation between self-care engagement and development of compassion fatigue was found. Individuals who engaged more in self-care activities were found to have lowered self-report of compassion fatigue compared to those who were less involved. The role of self-care in prevention of compassion fatigue can therefore not be ignored. As observed, self-care activities reduce one's vulnerability to compassion fatigue by acting both as a protective and a management factor. As a protective and management factor, engagement in self-care activities improves an individual's wellbeing. Physical activities prevent development of health conditions such as arthritis and heart diseases and boosts mental functioning among other benefits. Physical activities are also used in both prevention and management of stress. Spiritual self-care helps one find meaning of life as well as finding a place in service to mankind which provides life fulfillment. These benefits are applicable to all other methods of self-care discussed in this study.

\section{Discussion of Findings on the Association of Self Care and Compassion Fatigue}

The study objective aimed at determining the role of self-care as a protective factor against development of compassion fatigue. Correlation analysis of self-care and compassion fatigue done in this study revealed a significant negative correlation between compassion fatigue and self-care engagement. These findings suggest that compassion 
fatigue scores reduce as individual involvement in self-care increases. Increased self-awareness on the impact that caring for the suffering would lead to can increase the appreciation that the role that self-care would play in improving one's wellbeing which will in turn reduce compassion fatigue scores. Further, engagement in self-care activities was noted to increase with years of experience and reached the highest peak among respondents in the 11-15 years category. As the amount of self-care increased with experience, compassion fatigue scores were found to drop therefore strengthening the argument that self-care is a protective factor to development of compassion fatigue.

A comparison of self-care scores between the two work settings also showed that participants in the more traumatizing work settings engaged less in self-care activities as compared to those in the less traumatizing work settings. This is further evidenced by the compassion fatigue trend in the two work settings where high scores were registered in the high traumatizing work settings. The high compassion fatigue scores can therefore be attributed to high exposure to traumatic materials and lack of adequate self-care among the health care professional.

Further, the overall contribution of the construct self-care in the regression model was significantly high. It would therefore be prudent to confidently assert that if health care professionals increased their engagement in self-care activities, compassion fatigue would reduce considerably which would have a positive impact in both the individuals' lives and their life in the world of work.

\section{Conclusion}

Correlation results of the association between self-care and compassion fatigue was -0.766 . The implication here is that as participants increase their engagement in self-care, compassion fatigue scores reduce. These negative findings lead to a conclusion that self-care is a protective factor for the development of compassion fatigue among health care professionals.

\section{Recommendations}

Regular training on self-care that encompasses all the six subsets of self-care (Physical, Spiritual, Emotional, Psychological, and Workplace Balance) should be introduced in the work place. This will help in both preventing compassion fatigue and managing it whenever it occurs. Participation in self-care activities is set to improve the health professionals' wellbeing, boost their moral which will in turn lead to improved productivity. This can be done through Continuous Profession Development (CPD) and should be tailored to suit these work settings

\section{References}

i. Bowen, N. V. (2010). Common characteristics of compassionate mental health counselors: A Qualitative Study. (Doctoral thesis Ohio State University). Retrieved from https://etd.ohiolink.edu/ap/10?0::NO:10:P10_ACCESSION_NUM

ii. Bukibinga, P. (2012). The experience of job engagement and self-care among Ugandan nurses and midwives. (Doctoral thesis. University of Bergen). Retrieved from https://bora.uib.no/bitstream/.

iii. Fraenkel, J. \& Wallen, N., (2009). How to Design and Evaluate Research in Education. 7th ed. New York: McGraw Hill.

iv. Gardner III, K.E. (2014). Differences in perceptions of compassion fatigue, compassion satisfaction, and burnout among nursing faculty. (Unpublished Doctoral thesis Indiana University of Pennsylvania). Retrieved from: https://dspace.iup.edu/bitstream/handle/

v. Hunsaker, H. S, (2013). Identifying Factors That Influence Compassion Fatigue, Burnout, and Compassion Satisfaction in Emergency Department Nurses. Retrieved fromcontentdm.uvu.edu/cdm/ref/collection/UVU Theses/id/620.

vi. Kearney, M. K., Weininger, R. B.\& Vachon, M.L.S. (2009). Self-care of physicians caring for patients at the end-oflife. Retrieved: www.gpscbc.ca/.../Jama\%20Article-Self\%20Care\%20of\%20Physicians.p...

vii. Kiwol. S, Youngsook. S \& Hee J. K (2012). Relationships between Compassion Fatigue, Burnout, and Turnover Intention in Korean Hospital Nurses. J Korean Acad Nurses Vol. 42 No 7 December 2012. Retrieved from www.kan.or.kr/kor/shop_sun/files/.../mm__201212311831010.pdf

viii. Nassiuma, D. K. (2000). Survey sampling: Theory and methods. Njoro, Kenya: Egerton University

ix. Ndung'u, O. W. (2008). The role of career guidance and counseling in career awareness and planning among public secondary school students in Kenya. (Doctoral Thesis, Egerton University). Retrieved: rlibrary.egerton.ac.ke/.../the\%20role\%20of\%20career\%20gui.

x. Saakvitne, K.W. \& Pearlman, L.A. (1996). The Self-Care Assessment Worksheet. Traumatic Stress Institute/Center for Adult \& Adolescent Psychotherapy LLC. London. Retrieved from https://www.ecu.edu/.../Wellness.

xi. Smit, J. (2006). The influence of coping and stressors on burnout and compassion fatigue among health care professionals. (Doctoral thesis university of the Free State). Retrieved from .etd.uovs.ac.za/ETDdb/theses/available/etd-09252007.../SmitJ.pdf

xii. Stamm, B. H. (2009). Professional quality of life: Compassion satisfaction and fatigue. Version 5 (ProQOL). Retrieved from www.ProQOL.org

xiii. Star, K. L. (2013). The Relationship between Self-Care Practices, Burnout, Compassion Fatigue, and Compassion Satisfaction among Professional Counselors and Counselors-In-Training. UMI Dissertation Publishing, Microform Edition. USA. Retrieved from https://etd.ohiolink.edu/ap/ 